\title{
Análisis del experimento de Millikan
}

\author{
Héctor G. Riveros \\ Instituto de Física UNAM \\ Received 12 February 2020; accepted 6 June 2020
}

\begin{abstract}
Este experimento demostró la cuantización de la carga y midió la magnitud de la carga del electrón. Dicho experimento se repite en laboratorios escolares, aunque causa muchas decepciones; sin embargo es interesante interpretar los datos presentados por el científico en su recepción del premio Nobel y en un artículo en Physical Review. Millikan paso muchos años perfeccionando el experimento, para dismunuir las corrientes de convección y el tamaño de las gotas de aceite usado para reducir la evaporación de la gota. Sus mejores datos corresponden a gotas tan pequeñas que tardaban 120 segundos en caer $1.303 \mathrm{~cm}$, las cuales pueden incrementarse con 1 a 4 electrones. En laboratorios escolares pocos logran medir la misma gota en varios ciclos de subir y bajar, en consecuencia se reportan datos increíbles y quienes experimentan se sienten frustrados con sus resultados. Medir la velocidad de caída permite calcular la cantidad de electrones que necesita su gota para vencer la gravedad.
\end{abstract}

Descriptores: Millikan; viscosidad; interpretación.

This experiment demonstrated the quantization of the charge and measured the magnitude of the charge of the electron. It is an experiment that is repeated in school laboratories, causing many disappointments; although it is interesting to interpret the data presented at his Nobel Prize reception and in an article in Physical Review. Millikan spent many years refining the experiment, reducing convection currents and the size of the oil droplets, used to reduce evaporation from the droplet. His best data correspond to drops so small that it took 120 seconds to fall $1,303 \mathrm{~cm}$, which can be made to rise with 1 to 4 electrons. In school laboratories, few manage to measure the same drop in several cycles of up and down, which makes them report incredible data and feel frustrated with their results. Measuring the rate of fall allows you to calculate how many electrons your droplet needs to overcome gravity.

Keywords: Millikan; viscosity; interpretation.

PACS: 41.20.Cv; 01.40.-d

\section{Introducción}

En 1897, J.J. Thomson descubrió el electrón como partícula y midió la relación carga $/$ masa $=q / m=1.756 \times 10^{11} \mathrm{C} / \mathrm{kg}$. Esto fomentó la necesidad de medir su carga. Por esta razón R.A. Millikan diseñó un experimento para medir su carga $e$ y lo perfeccionó durante varios años. Sus mejores datos los presentó en su discurso Nobel [1], con una gota que tardaba en caer 120 segundos en una distancia de $1.3 \mathrm{~cm}$. Anteriormente, había publicado [2] otras gotas con tiempos de caída de 12 a 19 segundos. Demostró la cuantización de la carga y midió el valor de la carga de un electrón $e$. Este experimento clásico se hace en miles de laboratorios en universidades de todo el mundo. Melissinos [3] lo describe en su libro, y varios artículos sugieren formas de mejorar los experimentos estudiantiles. Kapusta [4] sugiere, mediante el cálculo de la propagación de errores, que los mejores resultados se obtienen para tiempos cercanos a 10 segundos, sin embargo, no advierte que eso requiere varios electrones en la gota. La utilización de video digital [5] mejora la precisión en la medida de los tiempos. Heald [6] propone medir el tiempo de caída y el voltaje para mantener flotando a la gota en el aire. Jones [7] propone usar una computadora para interpretar rápidamente los datos y calcular el radio de la gota y su carga. Halyo et al., [8] hacen una búsqueda sistemática de cargas fraccionarias usando los recursos modernos.
DOI: https://doi.org/10.31349/RevMexFisE.17.236

\section{Experimento}

El experimento consiste en dejar caer gotas cargadas entre dos placas metálicas conectadas a una fuente de voltaje. Sin aplicar voltaje, las gotas caen con la velocidad terminal calculada asumiendo una fricción viscosa de Stokes, de manera que la fricción cancela su peso:

$$
m g=6 \pi \eta a V_{c}=c V_{c}
$$

en donde $m$ es la masa, $g$ es la aceleración de la gravedad, $\eta$ es la viscosidad, $a$ es el radio de la gota, $c$ es una constante y $V_{c}$ es la velocidad de caída. Al aplicar el voltaje, aparece una fuerza eléctrica $q E$ que la sube hasta recuperar su altura original. Las fuerzas ahora son:

$$
q E=m g=6 \pi \eta a V_{s}=c V_{s}
$$

en donde $q$ es la carga eléctrica, $E$ es el campo eléctrico y $V_{s}$ es la velocidad terminal de subida. Al usar la constante $c$ para la fricción, se evita usar la ley de Stokes; así cualquiera que sea la fricción, la carga eléctrica es proporcional a la suma de velocidades $V_{c}+V_{s}$.

Al combinar 1 y 2 y despejar $q$ se obtiene: 


$$
\begin{aligned}
q & =\left(\frac{6 \pi \eta a}{E}\right)\left(V_{c}+V_{s}\right) \\
& =k\left(V_{c}+V_{s}\right)=\frac{c\left(V_{c}+V_{s}\right)}{E},
\end{aligned}
$$

en donde la constante $K=c / E$ depende de la viscosidad $\eta$, el radio a y el campo eléctrico E. Millikan propone que para gotas de tamaño comparable al camino libre medio 1 de las moléculas del aire es necesario usar una viscosidad efectiva calculada como:

$$
\text { viscosidad efectiva }=\frac{\eta}{(1+A l / a)}=\frac{\eta}{(1+b / p a)},
$$

en donde $A$ y $b$ son constantes y $p$ es la presión atmosférica.

Al escribir en la Ec. (1) la masa como volumen por densidad medida en el aire, se puede despejar el radio de la gota como:

$$
r=3\left(\frac{\eta V_{c}}{2 \rho g}\right)^{1 / 2}
$$

en donde aparece la viscosidad y es necesario calcularla considerando la viscosidad efectiva.

El experimento comienza al producir una nube de gotitas que pasan por un pequeño agujero en la placa superior. Se cargan eléctricamente por fricción o se bombardean con Rayos X o luz ultravioleta. Se observan con un microscopio enfocado en su trayectoria y se mide el tiempo que tardan en caer entre dos marcas. Al encender el campo eléctrico se mide el tiempo que tarda en pasar las mismas marcas. La temperatura entre las placas se controlaba con una precisión de $0.02^{\circ} \mathrm{C}$ para reducir la convección en el aire. En su discurso de aceptación del premio Nobel, Millikan expuso los datos de sus mejores resultados, presentados en la Tabla I [1].

\begin{tabular}{ccccc}
\hline \hline TABLA I. & & & & \\
\hline$T$ caída s & $T$ subida s & $V$ sub m/s & $V_{c}+V_{s} \mathrm{~m} / \mathrm{s}$ & $-a+\mathrm{m} / \mathrm{s}$ \\
\hline 120.8 & 26.2 & 0.000497 & 0.000606 & 0.000300 \\
121 & 11.9 & 0.001095 & 0.001203 & 0.000300 \\
121.2 & 16.5 & 0.000790 & 0.000898 & 0.000302 \\
120.1 & 16.3 & 0.000799 & 0.000908 & 0.000598 \\
120.2 & 26.4 & 0.000494 & 0.000602 & 0.000602 \\
119.8 & 67.4 & 0.000193 & 0.000302 & 0.000602 \\
120.1 & 26.6 & 0.000490 & 0.000598 & 0.000606 \\
- & 16.6 & 0.000785 & 0.000893 & 0.000893 \\
120.2 & 16.6 & 0.000785 & 0.000893 & 0.000893 \\
- & 16.4 & 0.000795 & 0.000903 & 0.000898 \\
120.2 & 68 & 0.000192 & 0.000300 & 0.000903 \\
119.9 & 67.8 & 0.000192 & 0.000300 & 0.000908 \\
- & 26.4 & 0.000494 & 0.000602 & 0.001203 \\
\hline Promedio & tiempos & de caída & 120.35 & \\
\hline
\end{tabular}

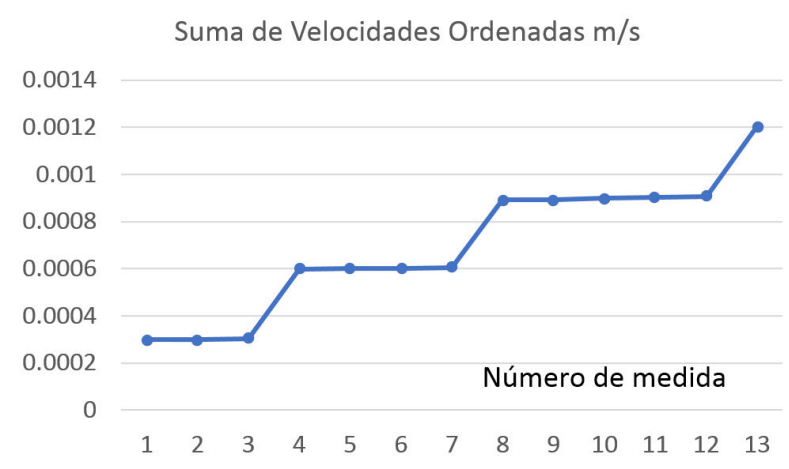

FIGURA 1. Suma de las velocidades de caída y subida medidas por Millikan. La suma de las velocidades son múltiplos enteros (2, 3 y 4) de la suma de velocidades más pequeña.

La Tabla I muestra en las columnas 2 e $i$ los valores de Millikan, mientras que las otras columnas fueron calculadas por el autor. Nótese la constancia de los tiempos de caída y el hecho de que tarda dos minutos en caer un centímetro. La constancia indica la ausencia de turbulencia en la cavidad y el tiempo tan largo sugiere lo pequeño de la gota. La velocidad de caída $V_{c}$ en la suma de velocidades se tomó como su valor promedio. La quinta columna muestra la suma de velocidades ordenadas de menor a mayor. La Fig. 1 presenta la suma de las velocidades $V_{c}+V_{s}$ ordenadas de menor a mayor en $\mathrm{m} / \mathrm{s}$.

Esta grafica demuestra la cuantización de la carga. Millikan menciona la distancia recorrida $=1.303 \mathrm{~cm}$, en tanto que el campo eléctrico es cerca de $6000 \mathrm{~V} / \mathrm{cm}$ y no senñala la densidad del aceite ni la temperatura del aire. Esto impide obtener el valor de la carga del electrón, sin embargo, Millikan la calculó como $e=(4.777 \pm 0.005) \times 10^{-10}$ unidades de carga electrostática. Al dividir cada suma de velocidades entre 2,3 o 4 se obtienen 4 valores que se combinan como sigue $V_{c}+V_{s}=(3.005 \pm 0.006) \times 10^{-4} \mathrm{~m} / \mathrm{s}$, para obtener el valor promedio para un electrón. Es posible calcular la constante $k$ usando el valor actual de $e$ y se obtiene $k=(5.33 \pm 0.01) \times 10^{-16} \mathrm{Cs} / \mathrm{m}$. Si se utilizan la densidad y temperatura de otra gota para calcular (3), se requiere que la viscosidad (4) se divida entre 1.045 para obtener la $k$ medida. Esta es la corrección de viscosidad efectiva. En la Tabla II se presentan datos de otra gota [2] tomados de la Tabla IV de su artículo. Se recopilaron los datos del tiempo de caída en la columna 1 , los tiempos de subida medidos con un cronómetro con resolución de $=.1$ segundos en la columna 2 y los tiempos medidos con otro cronómetro con resolución de 0,002 segundos en la columna 3. En su primera columna, el penúltimo renglón tiene el promedio de los tiempos de caída, mientras en la cuarta columna muestra el valor de la velocidad $V_{o}$, que sería la suma $V_{c}+V_{s}$ de una gota con carga de un electrón, y en la sexta columna aparece la velocidad de caída promedio utilizada por Millikan. El último renglón presenta la desviación normal del tiempo promedio de caída. Este experimento le tomó 45 minutos.

Al comparar las columnas con los tiempos de subida, se aprecia que en muchos casos el redondeo del cronómetro de precisión coincide con la lectura del cronómetro normal; la 


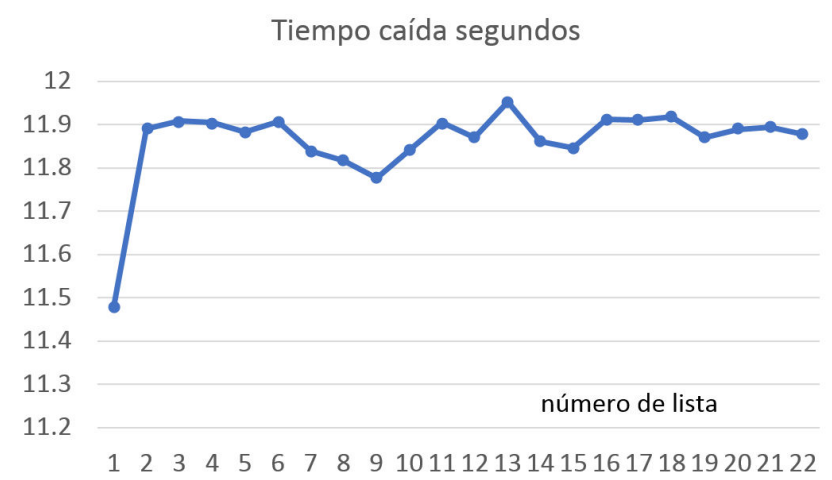

FIgURA 2. Tiempos de caída medidos, Millikan quito de su promedio el primer valor reportando 11.88 segundos como tiempo promedio.

mayor diferencia es 0.5 segundos. Las velocidades de subida se calculan dividiendo la distancia recorrida $=0.1021 \mathrm{~m}$ entre el tiempo promedio de caída. Las columnas 4 y 5 tienen las velocidades de subida $V_{s}$ dividiendo la distancia recorrida entre el tiempo de subida con cada cronómetro. Las columnas 6 y 7 presentan la suma de velocidades para los cronómetros 1 y 2 . Las columnas 8 y 9 muestran la división de la suma de ve- locidades entre la velocidad $V_{o}=5.49 \mathrm{E}-5 \mathrm{~m} / \mathrm{s}$. Aqui se aprecia que son números enteros desde 16 a 24 , de manera que esta gota requiere un mínimo de 16 electrones para vencer a la gravedad. La igualdad de los números de las columnas 8 y 9 demuestra que es suficiente un cronómetro para estas medidas. Millikan toma como tiempo de caída promedio 11.880 segundos, que difiere del valor calculado usando sus datos. La Fig. 2 muestra la gráfica de los tiempos de caída.

Al medir el cruce de la primera marca se introduce un retraso debido a la rapidez de la respuesta del dedo, pero al medir el cruce de la segunda marca se introduce un retraso casi igual. La medición del período de un péndulo con un cronómetro manual se considera normal con una precisión de un decimo de segundo, al medir una sola oscilación. Actualmente, medir milésimas de segundo requiere cámaras de muchos cuadros por segundo. Al tomar el tiempo promedio de caída se calcula una velocidad $V_{c}=0.0008594 \mathrm{~m} / \mathrm{s}$, que se suma con las velocidades de subida $V_{s}$ para obtener la suma $V_{c}+V_{s}$. Se determinó que el valor $V_{o}=5.49 \times 10^{-5}$ permite encontrar valores enteros para el cociente $\left(V_{c}+V_{s}\right) / V_{o}$, como lo muestra la Fig. 3.

\begin{tabular}{|c|c|c|c|c|c|c|c|c|}
\hline \multicolumn{9}{|l|}{ TABLA II. } \\
\hline$T$ caída s & $T$ subida1 $\mathrm{s}$ & $T$ subida2 $\mathrm{s}$ & Vel. subida $1 \mathrm{~m} / \mathrm{s}$ & Vel. subida $2 \mathrm{~m} / \mathrm{s}$ & $V_{c}+V_{s} \mathrm{~m} / \mathrm{s} 1$ & $V_{c}+V_{s} \mathrm{~m} / \mathrm{s} 2(V$ & $\left.+V_{s}\right) / V_{o} 1\left(V_{c}\right.$ & $\left.+V_{s}\right) / V_{o} 1$ \\
\hline 11.48 & 80.2 & 80.708 & 0.000127307 & 0.000126505 & $9.87 \mathrm{E}-04$ & $9.86 \mathrm{E}-0.4$ & 17.97 & 17.96 \\
\hline 11.89 & 22.4 & 22.366 & 0.00455804 & 0.000456496 & $1.32 \mathrm{E}-03$ & $1.32 \mathrm{E}-03$ & 23.96 & 23.97 \\
\hline 11.908 & & 22.39 & & 0.000456007 & & $1.32 \mathrm{E}-03$ & 0.00 & 23.96 \\
\hline 11.904 & 22.4 & 22.368 & 0.000455804 & 0.000456456 & $1.32 \mathrm{E}-03$ & $1.32 \mathrm{E}-03$ & 23.96 & 23.97 \\
\hline 11.882 & 140.4 & 140.565 & $7.27208 \mathrm{E}-05$ & 7.26354E-05 & $9.32 \mathrm{E}-04$ & $9.32 \mathrm{E}-04$ & 16.98 & 16.98 \\
\hline 11.906 & 79.6 & 79.6 & 0.000128266 & 0.000128266 & $9.88 \mathrm{E}-04$ & $9.88 \mathrm{E}-04$ & 17.99 & 17.99 \\
\hline 11.838 & & 34.748 & & 0.00029383 & & $1.15 \mathrm{E}-03$ & & 21.01 \\
\hline 11.816 & & 34.762 & & 0.000293712 & & $1.15 \mathrm{E}-03$ & & 21.00 \\
\hline 11.776 & & 34.846 & & 0.000293004 & & $1.15 \mathrm{E}-03$ & & 20.99 \\
\hline 11.84 & & 29.286 & & 0.000348631 & & $1.21 \mathrm{E}-03$ & & 22.00 \\
\hline 11.904 & 29.3 & 29.236 & 0.000348464 & 0.000349227 & $1.21 \mathrm{E}-03$ & $1.21 \mathrm{E}-03$ & 22.00 & 22.02 \\
\hline 11.87 & 137.4 & 137.308 & $7.43086 \mathrm{E}-05$ & 7.43584E-05 & $9.34 \mathrm{E}-04$ & $9.34 \mathrm{E}-04$ & 17.01 & 17.01 \\
\hline 11.952 & 34.9 & 34.638 & 0.00029255 & 0.000294763 & $1.15 \mathrm{E}-03$ & $1.15 \mathrm{E}-03$ & 20.98 & 21.02 \\
\hline \multicolumn{9}{|l|}{11.86} \\
\hline 11.846 & & 22.104 & & 0.000461907 & & $1.32 \mathrm{E}-03$ & & 24.07 \\
\hline 11.912 & & 22.268 & & 0.000458505 & & $1.32 \mathrm{E}-03$ & & 24.01 \\
\hline 11.91 & & 500.1 & & $2.04159 \mathrm{E}-05$ & & $8.80 \mathrm{E}-04$ & & 16.03 \\
\hline 11.918 & & 19.704 & & 0.000518169 & & $1.38 \mathrm{E}-03$ & & 25.09 \\
\hline 11.87 & & 19.668 & & 0.000519117 & & $1.38 \mathrm{E}-03$ & & 25.11 \\
\hline 11.888 & & 77.63 & & 0.000131521 & & $9.91 \mathrm{E}-04$ & & 18.05 \\
\hline 11.894 & 77.6 & 77.86 & 0.000131572 & 0000131133 & $9.91 \mathrm{E}-04$ & 9.91E-04 & 18.05 & 18.04 \\
\hline 11.878 & 42.6 & 42.302 & 0.000239671 & 0.00024136 & 1.10E-03 & $1.10 \mathrm{E}-03$ & 20.02 & 20.05 \\
\hline 11.861 & & $V_{o} \mathrm{~m} / \mathrm{s}$ & 5.49E-05 & $V_{c} \mathrm{~m} / \mathrm{s}$ & 0.008594276 & & & \\
\hline 0.093508849 & & & & & & & & \\
\hline
\end{tabular}


$(\mathrm{Vc}+\mathrm{VS}) /$ Vo O Número de electrones

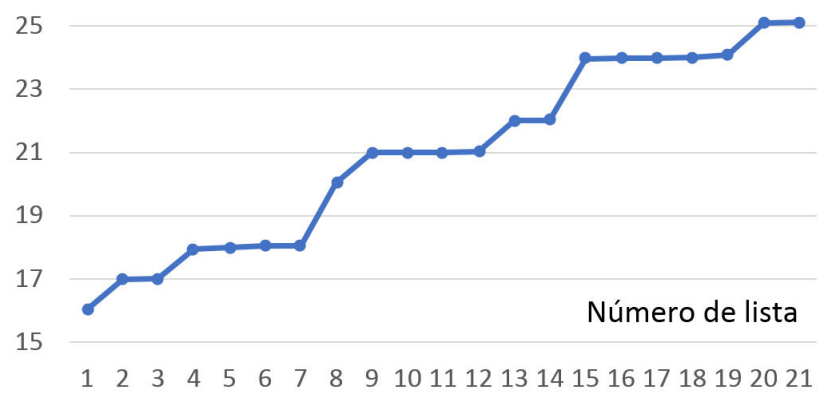

FIgURA 3. Los valores de la suma $V_{c}+V_{s}$ son múltiplos enteros de $V_{o}$ e indican el número de electrones en la gota. Si la gota tiene mucha masa, requiere muchos electrones para vencer a su peso.

La carga en la gota es proporcional a esta suma: $q=$ $k\left(V_{c}+V_{s}\right)$ Ec. (3). La constante de proporcionalidad es $k=(6 \pi \eta a / E)$. Al calcular $K$ usando los valores MKS se obtiene: $K=3.03 \times 10^{-15} \mathrm{Cs} / \mathrm{m}$ y se puede determinar la carga en cada gota, pero no se obtienen múltiplos enteros de $e=1.602 \times 10^{-19} \mathrm{C}$. Al usar $e=1.602 \times 10^{-19}$ $\mathrm{C} / 5.49 \times 10^{-5}$ se obtiene $k=2.92 \times 10^{-15} \mathrm{Cs} / \mathrm{m}$, lo cual justifica la necesidad de usar una viscosidad efectiva. Los laboratorios escolares con cronómetros de 0.01 segundos de resolución, pueden medir la carga en las gotas de aceite, siempre y cuando tengan gotas suficientemente pequeñas para no requerir muchos electrones.

\section{Masa de la gota y el tiempo de caída}

El tamaño mínimo de la gota depende del atomizador de las gotas. Para medir conviene hacerlo con la gota más lenta. Al medir su velocidad de caída se puede calcular el radio, usando la viscosidad a la temperatura adecuada, no obstante, su valor efectivo la reduce un poco. Con el radio y la densidad se obtiene la masa. Si se conoce el peso en Newtons se puede calcular la fuerza eléctrica y estimar el número mínimo de electrones necesario para levantar a la gota. La Tabla III muestra un ejemplo calculado para que el estudiante tenga una idea de cuántos electrones requiere su gota para subir.

Los datos del experimento son: distancia de caída $=0.013$ $\mathrm{m}$, densidad de la gota $=900 \mathrm{~kg} / \mathrm{m} 3$, viscosidad del aire $=$ $1.82 \mathrm{E}-5$ Pas, $\mathrm{g}=9.8 \mathrm{~m} / \mathrm{s}^{2}$, voltaje $=4000$ volts, separación entre placas $=0.016 \mathrm{~m}$, campo eléctrico $=250000 \mathrm{v} / \mathrm{m}$.

La distancia de caída es la usada por Millikan, pero se calculó con un voltaje menor. El radio se determina con la Ec. (5) y la última columna es el número mínimo de electrones necesarios para vencer el peso de la gota. Para tiempos de caída menores a 10 segundos crece mucho el número mínimo de electrones necesario para vencer la fuerza de gravedad. Con estos datos se puede escoger el voltaje más adecuado o cancelar el experimento. Tal como lo hizo Milikan, es conveniente medir varias veces las velocidades para estimar la incertidumbre en la carga y corroborar su presencia en múltiplos enteros.

Recomendación. La interpretación de los datos de Millikan es un excelente ejercicio para los estudiantes y permite entender la razón por la cual los experimentos escolares con equipos con tiempos de caída menores a 100 segundos son complicados de interpretar, debido al número de electrones necesarios para vencer el peso de gotas relativamente grandes. Medir el tiempo de caída en su laboratorio y la lectura de este artículo, ayudará a interpretar sus datos, que generalmente presentan tiempos de caída muy cortos.

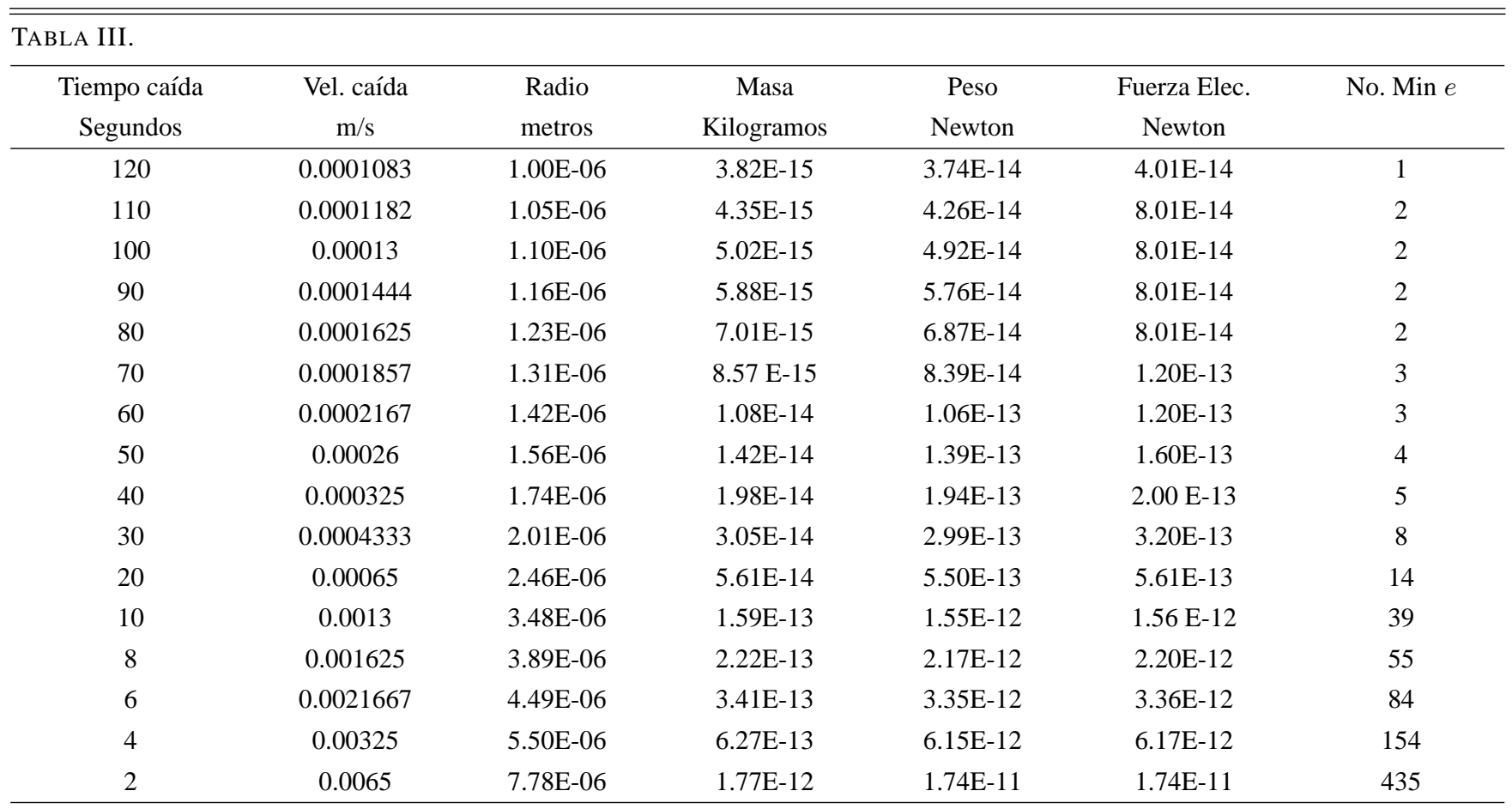


1. Nobel Foundation, Nobel Lectures in Physics 1922-1941, Elsevier, pag. 49.

2. R. A. Millikan, "On the elementary electric charge and the Avogadro constant”, Phys. Rev. (Series II) 2 (Aug. 1913) 109-143 . https://doi.org/10.1103/PhysRev.2.109.

3. A. Melissinos, Experiments in Modern Physics, Academic Press, New York, 1966, pag. 2-7. American Journal of Physics 73 (2005) 789 ; doi:10.1119/1.1848112

4. J. I Kapuska, Best measuring time for a Millikan oil drop experiment, American Journal of Physics 43 (1975) 799; doi: $10.1119 / 1.9710 \mathrm{KJ}$

5. K.J. Silva and J.C. Mahendra, "Digital video microscopy in the Millikan oil-drop experiment",
6. M.A. Heald, Millikan Oil-Drop Experiment in the Introductory Laboratory, American Journal of Physics 42 (1974) 244; doi:10.1119/1.1987656

7. Ray C. Jones, "The Millikan oil-drop experiment: Making it worthwhile", Am. J. Phys. 63 (Nov. 1995) 970-977 https: //doi.org/10.1119/1.18001

8. V. Halyo et al, Search for Free Fractional Electric Charge Elementary Particles, SLAC-PUB-8283, Oct 1999, arXiv.org > hep-ex > arXiv:hep-ex/9910064

9. Viscosidad del aire: www.engineersedge.com/ physics/viscosity of air dynamic and kinematic 14483.htm leído 2/12/2019 